\title{
The power of universal pictures
}

\author{
The sky map presented by the Dark Energy Survey showcases the power of images to reach scientists \\ and the wider public alike.
}

Maps of the cosmic microwave background (CMB) have become iconic representations of the early Universe, and the increasing resolution of these charts - from NASA's COBE and WMAP to ESA's Planck map are a testament to the impressive progress in observational cosmology. But when the Dark Energy Survey (DES) collaboration unveiled the analysis of their first year of data last month, many felt that a new era had started.

The DES, involving some 400 scientists from 26 institutions in seven countries, uses a record-setting 570-megapixel digital camera, mounted on the 4-metre Víctor M. Blanco Telescope at the Cerro Tololo Inter-American Observatory in Chile, as the primary instrument for looking at slight distortions of the shapes of distant galaxies due to weak gravitational lensing. Their first-year data alone contains information about 26 million galaxies, which has then been painstakingly assembled into a map of the projected density of dark matter (pictured). This mass-density map complements the CMB charts, which plot the energy density around 400,000 years after the Big Bang, an epoch where matter dominated. DES, in contrast, charts the structure at much later times, after gravitational pull and dark-energy push have done their work in shaping the Universe.

What makes the DES mass map so impressive is that its quality is reminiscent of the best CMB maps, produced by the Planck

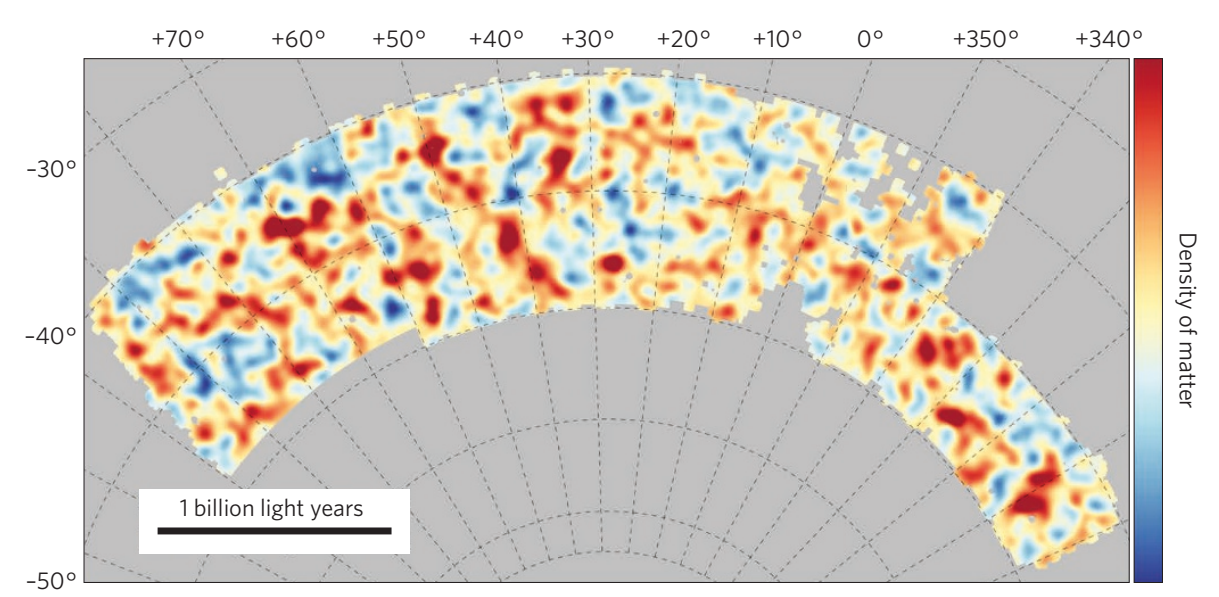

mission of 2009-2013. The DES results broadly support current notions about the matter and energy thought to be hidden in the dark sector, although the data did give rise to a number of questions that will keep researchers on their toes. More data is currently being analysed and acquired. The map now presented is based on data taken in the first observation season, from August 2013 to February 2014. Three more seasons' worth of data are being processed (and are publicly available one year after acquisition). The final scheduled season is currently getting underway. By the time all of the data are analysed, the DES map should grow three times larger than the current one.
But it is clear already that the map is en route to becoming an iconic image of our Universe, of the sort that CMB surveys have produced. As such, the DES has all the potential to capture the imagination of a broad public, including youngsters who will one day create the next generation of surveys. The Large Synoptic Survey Telescope (LSST) is scheduled to start collecting data in 2022 .

Reaching out to the public is also the mission of next month's Dark Matter Day (http://www.darkmatterday.com), not by accident set on 31 October, a day on which many parts of the world celebrate the 'unseen'. Don't miss the chance to hear, or talk, about the Universe's dark secrets.

\section{Physics students unite}

\section{The International Conference of Physics Students continues its remarkable tradition.}

Last month, 450 students from 44 different countries convened in the spectacular surroundings of the Campus Luigi Einaudi in Turin, Italy, at the International Conference of Physics Students (ICPS).

Invited speakers included Elena Aprile, an experimental particle physicist who founded the XENON dark-matter experiment operating at the Gran Sasso laboratory in central Italy, and Steven Cowley, a plasma physicist who, up until very recently, headed the UK Atomic Energy Authority. But most of the talks and presentations were given by students - not only a valuable opportunity for listeners to learn about new physics, but also a chance for presenters to practice the communication of their research.

The social and cultural calendar was another highlight: a rich itinerary of lab visits, tourism and social events such as the Nations Party (a celebration of the cuisine from all the participants' home countries) ensured the conference was also a wonderful and successful opportunity for cultural exchange.
Perhaps the most impressive aspect of the ICPS is how it is organized: this year it was the turn of the Italian branch of the International Association of Physics Students, the Associazione Italiana Studenti di Fisica, to secure its funding and coordination. Yet teams of dedicated students have displayed this initiative every year since 1986, when the first ICPS was held at the tail-end of the Cold War in Budapest, Hungary.

ICPS 2018 will be held in Helsinki, Finland, next August. Mark your calendars. $\square$ 